\title{
Single-objective selective-volume illumination microscopy enables high-contrast light-field imaging
}

$\underline{K e v i n ~ K e o m a n e e-D i z o n ~}^{1}{ }$, Sara Madaan ${ }^{1,2}$, Matt Jones ${ }^{1}$, Chenyang Zhong $^{1}$, Anna Nadtochiy ${ }^{1}$, Peter Luu ${ }^{1}$, Scott Fraser ${ }^{1}$, Thai Truong ${ }^{1}$ ${ }^{1}$ University of Southern California, USA. ${ }^{2}$ Google Inc, USA

\section{Abstract Text}

Biological processes often depend on the tight spatiotemporal coordination between cells across tissue-level length scales, extending over hundreds of microns in 3D. Functional understanding of such processes would be greatly aided by imaging tools that offer the combined speed and sensitivity needed to observe 3D cellular dynamics without compromising the normal biology. Light-field microscopy (LFM) is a fast, synchronous 3D imaging technique that captures both the 2D spatial and 2D angular information of light emitted from the sample, permitting computational reconstruction of the signal from a full volume in a single snapshot [1]. While the diffraction of light imposes a tradeoff between spatial resolution and $z$-depth coverage, LFM has successfully demonstrated synchronous imaging of depths covering hundreds of microns at cellular resolution, and more recently has achieved depths covering several microns at subcellular resolution. The full potential of LFM, however, has been hampered by conventional wide-field illumination where the entirety of sample is lit up, often beyond the volume of interest, creating significant extraneous background that degrades the image contrast, especially for thick and/or densely-labeled samples.

Light-sheet microscopy (also known as selective-plane illumination microscopy; SPIM) achieves high image contrast by illuminating only the optical plane of interest. Inspired by SPIM, we recently introduced an improved light-field-based imaging approach, termed selectivevolume illumination microscopy (SVIM), where confining excitation to only the volume of interest reduces extraneous background, thereby 
sharpening contrast and effective resolution in thick ( $\geq 100 \mu \mathrm{m})$ living systems [2]. SVIM was implemented with two objective lenses: one to selectively illuminate the volume of interest, and a second objective, orthogonally aligned, to acquire the fluorescent light-field. This two-objective geometry requires that the sample be mounted within the mutual intersecting volume defined by the perpendicular objectives, complicating sample mounting and thus precluding widespread adoption.

We will present our latest development in SVIM that uses only one objective, eliminating the need for two orthogonally oriented objectives at the specimen, and greatly broadening its utility for biological research. By implementing an oblique one-photon illumination path or two-photon illumination, the volume of interest is selectively excited through the same objective used for highnumerical-aperture detection. This single-objective approach simplifies specimen mounting, yet still reduces extraneous background, resulting in improved contrast, resolution, and reconstruction quality over traditional LFM. Rapid synchronous imaging of multicellular volumes spanning hundreds of microns will be shown, including quantification of cellular structures and brainwide neural activity in larval zebrafish, as well as imaging of multiple specimens mounted in a standard multi-well plate. 